\title{
The Adhesion of Gold Electrodeposits
}

\section{A FUNDAMENTAL LAW AND ITS PRACTICAL ASPECTS}

\author{
M. A. M. Bakker
}

N. V. Philips, Eindhoven, Netherlands

Based upon the Pourbaix diagrams, the author has established the conditions that must be fulfilled to ensure good adhesion of an electrodeposited metal to its substrate. In this article he outlines his principle and describes its application to modern gold plating practice.

A high standard of adhesion is naturally required in the many applications of electrodeposited gold in electronic devices. While perfect adhesion is readily achieved on copper or its alloys, some difficulty may be experienced with the iron-based alloys containing nickel, cobalt or chromium that are now so frequently employed for lead frames and other components in semi-conductor technology.

Gold plating electrolytes are formulated mainly to ensure optimum characteristics in the deposit, and relatively little attention has been given to considerations of adhesion, particularly for critical applications in which a heat treatment process is required after plating. It can be shown, however, that a fundamental basis does exist for the attainment of good adhesion, dependent upon the properties of the metal substrate and the composition of the electrolyte.

\section{Theoretical Considerations}

According to the Pourbaix diagrams every metal immersed in an aqueous solution is in an active, a passive or an immune state, depending on the $\mathrm{pH}$ of the solution. In the active state the metal goes into solution according to the equation:

$$
\mathrm{Me} \rightleftharpoons \mathrm{Me}^{+}+\mathrm{e}
$$

until equilibrium is reached.

Trunsistor assemblies such as this have their headers and lead wires gold plated. The headers are usually made from a nickel-iron alloy, and it is here that special care must he taken to ensure perfect adhesion of the gold electrodeposit
If the metal is not noble there is the possibility that no equilibrium will be reached because hydrogen ions are discharged or, if ions of greater nobility are present in that solution, immersion plating takes place, i.e.

or

$$
\begin{aligned}
\mathrm{Me}+\mathrm{H}^{+} & \rightleftharpoons \mathrm{Me}^{+}+\frac{1}{2} \mathrm{H}_{2} \\
\mathrm{Me}_{1}+\mathrm{Me}_{2}{ }^{+} & \rightleftharpoons \mathrm{Me}_{1}{ }^{+}+\mathrm{Me}_{2}
\end{aligned}
$$

When the metal is connected to a rectifier and a potential is applied using another electrode, the metal remains active when it is made anodic. Even at high anodic potentials the metal turns passive,

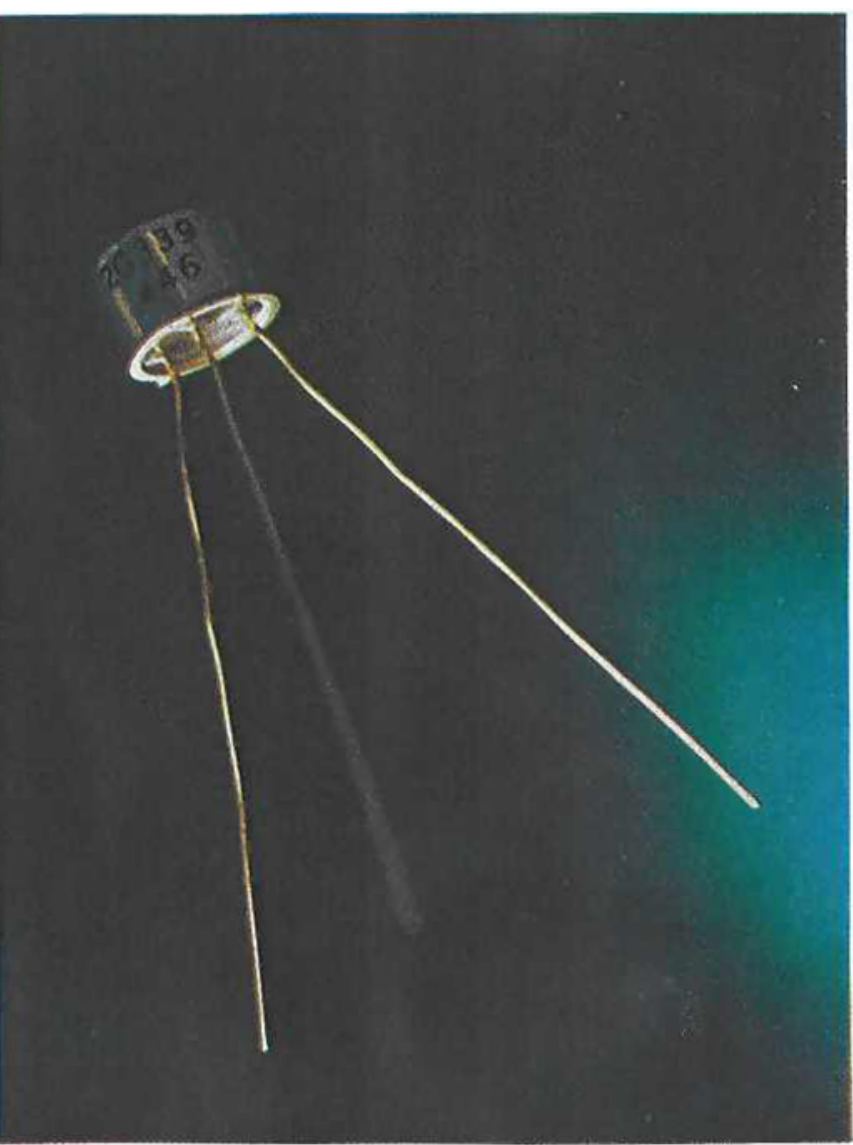




\section{Pourbaix Diagrams}

The graphical representation of the reactions between metals and aqueous solutions in the form of diagrams in which the equilibrium potential is plotted against $\mathrm{pH}$ for each reaction was conceived over 25 years ago by Professor Marcel Pourbaix of the Centre Belge d'Etude de la Corrosion (Cebelcor) of Brussels, and he and his colleagues have since completed diagrams for practically every element, published in their Atlas of Electrochemical Equilibria in Aqueous Solutions.

These diagrams, which must be regarded only as approximations, none the less compress a great deal of information into a small space. They can be of great usefulness in electrochemical studies both in showing the conditions in which the occurrence of an oxidation or a reduction reaction is possible from the energetic standpoint and in the prediction and interpretation of many phenomena in this field.

The example shown here is a much simplified form of Pourbaix's diagram for iron in equilibrium with its ions, oxides and water. It is upon the basis of this type of diagram, in conjunction with the electrode potentials of the metals concerned, that

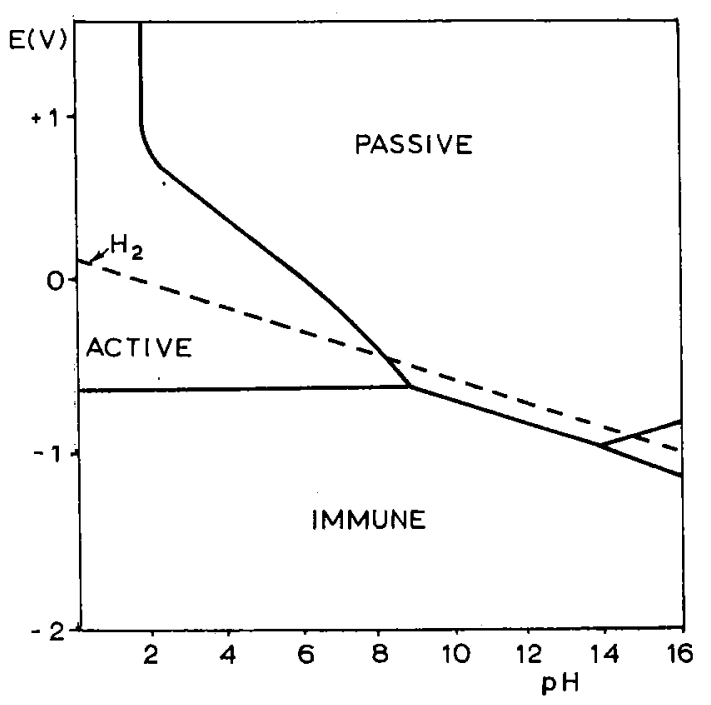

Mr Bakker has formulated the conditions that must be fulfilled in order to achieve good adhesion of any electrodeposit to its substrate and has made it possible to calculate the critical concentrations of certain constituents of the electrolyte at which adhesion becomes unsatisfactory. which means that an oxide film is formed on the metal surface:

$$
\mathrm{Me}+\mathrm{H}_{2} \mathrm{O} \rightleftharpoons \mathrm{MeO}+2 \mathrm{H}^{+}+2 \mathrm{e}
$$

The equilibrium potential of this reaction, the socalled "Flade Potential", is dependent only on the $\mathrm{pH}$ of the solution:

$$
\mathrm{E}_{\mathrm{F}}=\mathrm{E}_{\mathrm{O}}-0.59 \mathrm{pH}
$$

This means that at higher $\mathrm{pH}$ values in more alkaline solutions passivation occurs more rapidly and also more easily at lower potentials.

When, however, a cathode potential is applied the metal can be brought to its immune state. The metal is stable and no corrosive attack or surface oxide layers occur.

It can also be seen from the Pourbaix diagram that when a cathodic potential is applied the metal is not automatically in its immune state; the potential has to be more negative than the equilibrium potential, the partition line between the immune region on one side and the active and passive region on the other.

It can be stated then as a fundamental law that excellent adhesion can only be obtained if the basis metal is in the immune state dur. ing plating. No real metal-to-metal bond can be achieved if an oxide layer is present or when there is a tendency for the basis metal to go into solution.

\section{Practical Aspects}

What does all this mean for the plater, and especially for the gold plater, who nowadays has the choice between acid, neutral and alkaline gold plating baths?

To deposit gold from a plating bath it is only necessary to apply a potential just a little greater than the deposition potential of gold in that bath. This, however, does not imply that the metal to be plated is in its immune state at that potential. It can also be active, or even passive, depending on the Pourbaix diagram involved.

It is well-known that good adhesion is difficult to obtain with gold plating on to iron. When an acid gold plating bath is used, immersion plating occurs while iron is at that $\mathrm{pH}$ in its active state and poor adhesion results.

An alkaline or a neutral gold plating bath is a far better proposition, but the following phenomenon must be borne in mind. After the iron has been thoroughly degreased and etched it is well rinsed before entering the plating solution. If we look at the Pourbaix diagram for iron it can be seen that at pH 7 and without applying a potential (i.e. at zero potential) iron is in the passive state. Thus at the moment of entering the plating solution iron is still passive. A finite time is necessary to reduce the passive layer at the applied potential of the plating solution. This may be accomplished in a short time if the passive layer is thin and also when the potential is negative enough to bring the iron "deep" into the immune region of the diagram.

If hydrogen evolution takes place one cannot always say that the base metal is not passive but in 


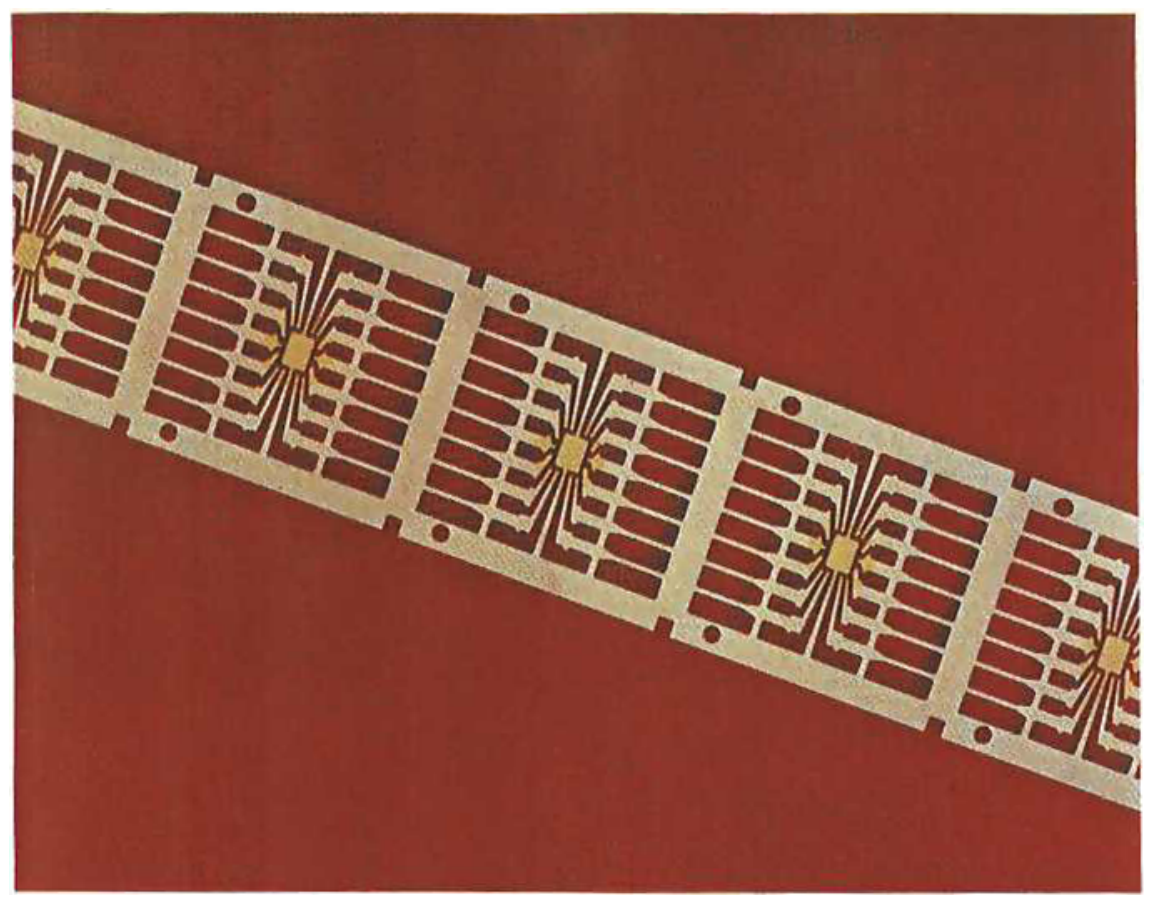

The selective area plating of lead frames, also fabricated from nickel-iron alloys containing colbalt or chromium, formerly presented considerable problems in achieving good adhesion of the gold electrodeposit. In addition to improved cleaning and etching procedures, the careful selection of bath eompositions and deposition potentials have now removed these difficulties

the immune state. Only when the hydrogen line, the dotted straight line in the Pourbaix diagram, is under the passive/immune partition line is it true. In all other cases hydrogen is set free at a passive metal surface!

Furthermore it must be realised that the Pourbaix diagram holds for a state of equilibrium, but there are kinetic effects, such as the dissolution rate of the passive layer, polarisation and overvoltage, so that the calculations are not always in complete agreement with the practical results. Also in the vicinity of the cathode the $\mathrm{pH}$ is usually higher than in the bulk of the plating solution, owing to the fact that hydrogen ions are discharged.

How can the problem of poor adhesion with gold plating baths be solved in the light of these factors?

The Pourbaix diagrams are determined, and can be used only as they stand. The deposition potentials, however, can generally be changed and can be made more negative by decreasing the gold ion concentration in the plating bath and, more especially, by decreasing the ion activity, which is normally done by complexing the ions. With gold plating baths it can be achieved by adding potassium cyanide, which makes the gold deposition potential so negative that most base metals, such as iron, nickel and their alloys, are in the immune state during plating. But the addition of cyanide is only possible in alkaline plating solutions because in acid solutions the cyanide will evaporate as hydrogen cyanide.

As in practice most commercial gold baths are used with a fixed composition and $\mathrm{pH}$ there is no possibility of changing the bath composition without endangering its special features. Therefore an alkaline gold strike bath has to be introduced when adhesion problems occur. A gold strike bath has a low gold content (less than $1 \mathrm{~g} / \mathrm{l}$ ) and a high cyanide content (over $100 \mathrm{~g} / 1$ ). After the gold strike is applied a quick water rinse must be carried out, after which the work must be plated as soon as possible in the gold plating bath normally used, which can be neutral or acid with brighteners or other alloying metal salts.

There are commercial gold strike baths available but usually they are of the acid type. This at once gives an improvement in adhesion because passivity of the base metal is prevented, but the deposition potential of the gold is not sufficiently negative to bring the base metal to the immune state. It has so far been impossible to complex the gold strongly enough in acid solutions.

In most cases it is possible to calculate the deposition potential of the gold from the equilibrium constant of the metal-chelating agent. From the Pourbaix diagram of the base metal it can then be seen whether adhesion difficulties can be expected at the pH of that gold plating bath and at the calculated deposition potential, and if so how the difficulties can be overcome.

If it is not pure iron that has to be plated but an iron-based alloy such as Kovar then one has to use the Pourbaix diagram of the least noble component, i.e. iron, although in practice the situation is more favourable owing to the fact that a part of the surface of the alloy is nickel or cobalt and that therefore this will act more nobly. 